\title{
Efficient synthesis of allylamines from a novel (E)-2,3-difunctionalized allyl bromide
}

\author{
Maha Ameur, Aïcha Arfaoui, and Hassen Amri* \\ Laboratory of Selective Organic Chemistry \& Biological Activity, Faculty of Science, \\ El Manar University, 2092 Tunis, Tunisia \\ E-mail: hassen.amri@fst.rnu.tn
}

DOI: http://dx.doi.org/10.3998/ark.5550190.0015.500

\begin{abstract}
We report a new procedure for highly stereoselective synthesis of difunctionalized allyl and vinyl bromides $\boldsymbol{E}-\mathbf{4}$ and $\boldsymbol{E}-\mathbf{5}$ via a tandem reaction of bromination-dehydrobromination of tetraethyl prop-2-ene-1,2-diyldiphosphonate in the presence of DBU in acetonitrile at room temperature. The coupling reaction of allyl bromide $\boldsymbol{E}-\mathbf{4}$ with various primary amines in methanol at $0{ }^{\circ} \mathrm{C}$, followed by $\mathrm{S}_{\mathrm{N}} 2$ ' reaction, provides a new family of tetraethyl 1-(alkylamino)prop-2-ene-1,2-diyldiphosphonates 6 in high yields.
\end{abstract}

Keywords: Diethyl 1-(hydroxymethyl) vinylphosphonate, tetraethyl prop-2-ene-1,2diyldiphosphonate, DBU, allyl bromide, allylamine

\section{Introduction}

Functinalized allyl $^{1-6}$ and vinyl bromides ${ }^{7-10}$ are often used as intermediates for the synthesis of biologically active compounds ${ }^{11-16}$ including natural products. ${ }^{17,18}$ Continuing with our efforts directed toward the development of new methodologies for the synthesis of functionalized brominated derivatives, ${ }^{19-24}$ we describe in this paper an efficient alternative for the preparation of a new family of allyl bromide $(\boldsymbol{E})-\mathbf{4}$ and vinyl bromide $(\boldsymbol{E})-5$ from tetraethyl prop-2-ene-1,2diyldiphosphonate 2 (Scheme 1) with complete stereoselectivity in favour of the $E$ configuration starting from inexpensive reagents. Allyl bromide $(\boldsymbol{E})-\mathbf{4}$ has been separated and then transformed in a one-step synthesis, to a new family of tetraethyl 1-(alkylamino)prop-2-ene-1,2diyldiphosphonates 6. 

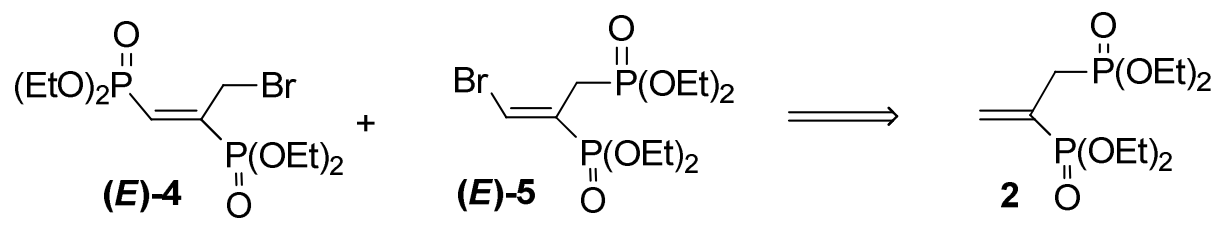

Scheme 1. Retrosynthetic analysis.

\section{Results and Discussion}

Our strategy began with the synthesis of functional allyl bromide 1 (90\%) from diethyl 1-(hydroxymethyl) vinylphosphonate ${ }^{25}$ using phosphorus tribromide as brominating agent $(0.5$ equiv.) in diethyl ether at $-10{ }^{\circ} \mathrm{C}$, followed by nucleophilic substitution of the obtained allyl bromide 1 by triethylphosphite ${ }^{26,27}$ (1 equiv.) at $80{ }^{\circ} \mathrm{C}$, to provide only tetraethyl prop-2-ene-1,2diyldiphosphonate $\mathbf{2}$ in quantitative yield (Scheme 2).

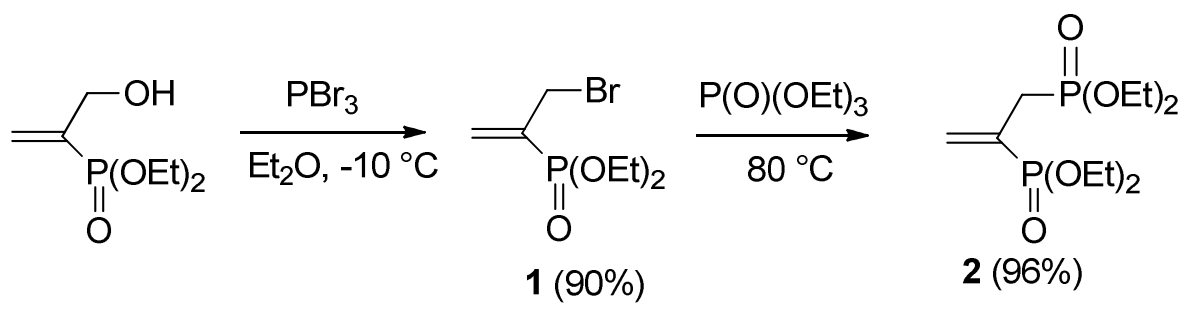

Scheme 2. Synthesis of tetraethyl prop-2-ene-1,2-diyldiphosphonate 2.

The addition of bromine in refluxing carbon tetrachloride to the vinyldiphosphonate 2 led to the corresponding dibromo compound $\mathbf{3}$ in $91 \%$ yield. In order to find optimal conditions for the synthesis of functionalized allyl bromide $\mathbf{4}$, we examined the dehydrobromination reaction of $\mathbf{3}$ at room temperature under various reaction conditions. As shown in Table 1, poor yields of compound 4 were obtained in the presence of $\mathrm{Et}_{3} \mathrm{~N}$ and $\mathrm{K}_{2} \mathrm{CO}_{3}$, while the use of DABCO was completely ineffective. However, the best result was obtained when the reaction was carried out in the presence of 1,8-diazabicyclo[5.4.0]undec-7-ene (DBU, 1.1 equiv.) in acetonitrile. It should be noted that the synthesis of allyl bromide $\mathbf{4}$ was always accompanied by the formation of its regioisomer $\mathbf{5}$, which was easily separable from the desired product $\mathbf{4}$ by silica gel column chromatography (Scheme 3).

Table 1. Effect of reaction conditions on the synthesis of functionalized bromides $\mathbf{4}$ and $\mathbf{5}$

\begin{tabular}{ccccc}
\hline Entry & Base & Solvent & Time (days) & Yield (\%) of (4/5) \\
\hline $\mathbf{1}$ & $\mathrm{DABCO}$ & $\mathrm{THF}$ & 10 & - \\
$\mathbf{2}$ & $\mathrm{Et}_{3} \mathrm{~N}$ & $\mathrm{CCl}_{4}$ & 15 & $16 / 4$ \\
$\mathbf{3}$ & $\mathrm{DBU}$ & $\mathrm{CH}_{3} \mathrm{CN}$ & 3 & $73 / 8$ \\
$\mathbf{4}$ & $\mathrm{K}_{2} \mathrm{CO}_{3}$ & $\mathrm{THF}$ & 3 & traces \\
\hline
\end{tabular}



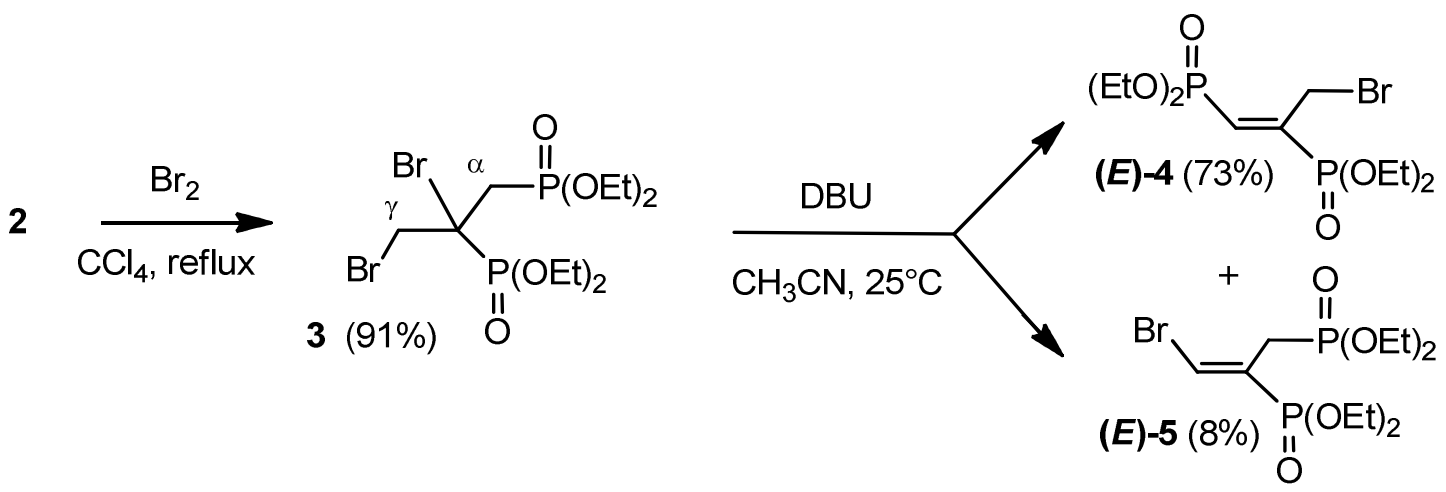

Scheme 3. Synthesis of allyl and vinyl bromides $(\boldsymbol{E})-\mathbf{4},(\boldsymbol{E})-\mathbf{5}$.

The formation of two brominated regioisomers $(\boldsymbol{E})-\mathbf{4}$ and $(\boldsymbol{E})-\mathbf{5}$ can be explained on the basis of the elimination reaction of hydrogen bromide due to the mobility of hydrogen atoms $\mathrm{H}_{\alpha}$ and $\mathrm{H}_{\gamma}$, while the inherent stereochemistry of the two regioisomers can be justified on the basis of the examination of the two possible conformational equilibria of $\mathbf{3}$ and the choice of the most stable conformer in harmony with both steric bulk and electronic effects. The configuration of each of allyl- and vinylbromides $(\boldsymbol{E}) \mathbf{- 4}$ and $(\boldsymbol{E})-\mathbf{5}$ was elucidated by two-dimensional NMR (NOESY). Indeed, the absence of correlation between the vinyl proton $(6.70 \mathrm{ppm})$ and the $\mathrm{CH}_{2} \mathrm{Br}$ protons (4.6 ppm) for the allyl bromide $(\boldsymbol{E})-\mathbf{4}$ and the ethylenic proton $(7.48 \mathrm{ppm})$ and the $\mathrm{CH}_{2} \mathrm{P}(\mathrm{O})(\mathrm{OEt})_{2}(3.04 \mathrm{ppm})$ in the case of vinyl bromide $(\boldsymbol{E})-\mathbf{5}$ were in favour of the geometry $(E)$ of the above regioisomers. It is also worth noting that there is good agreement between the experimental chemical shifts of vinylic protons of functionalized bromides $(\boldsymbol{E}) \mathbf{- 4}$ and $(\boldsymbol{E})-\mathbf{5}$ and those calculated by Pascual's formula ${ }^{28,29}$ (Scheme 4).

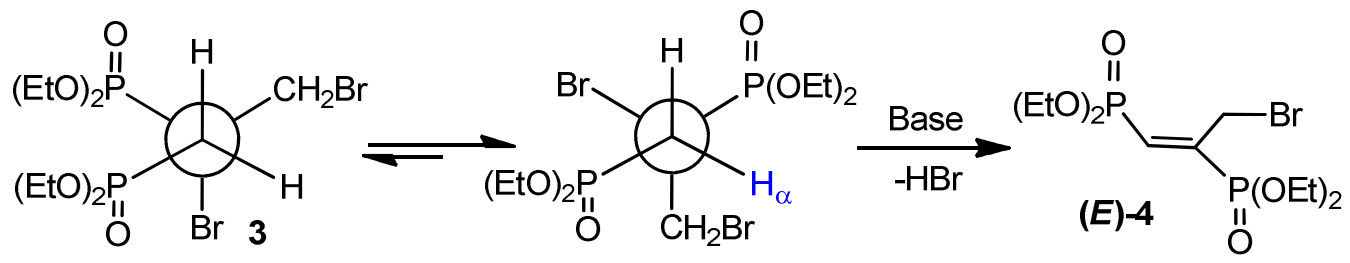<smiles>C=CC=CC</smiles>

Scheme 4. Allyl and vinyl bromides $(\boldsymbol{E})-\mathbf{4}$ and $(\boldsymbol{E})-\mathbf{5}$ in accordance with Newman projections of 3. 
In order to establish a chemical library reserved for the various functionalized allylamines published during the last decade by our research group ${ }^{30}$ and their use as basic skeletons of many biologically important substances ${ }^{31-37}$ and numerous natural products ${ }^{38-47}$, we focused our attention on the synthesis of a new family of allylamines $\mathbf{6}$. The best reaction conditions were obtained following the reaction of the electrophilic allyl bromide $(\boldsymbol{E})-\mathbf{4}$ with excess of monoalkylamines (2 equiv.) in methanol at $0{ }^{\circ} \mathrm{C}$ (entries 1-7). Due to the coexistence of the phosphoryl and bromomethyl groups on the same carbon atom, the intermediate $(\boldsymbol{E}) \mathbf{- 4}$ reacts regioselectively with amines in a clean $S_{N} 2$ ' reaction, to provide tetraethyl 1-(alkylamino)prop-2ene-1,2-diyldiphosphonates 6 with high purity and satisfactory yields (Scheme 5, Table 2). Furthermore, the reaction time and the overall yields have not been affected by the donor effects (entries 1-3) or withdrawing effects (entries 4-7) of the alkyl groups.
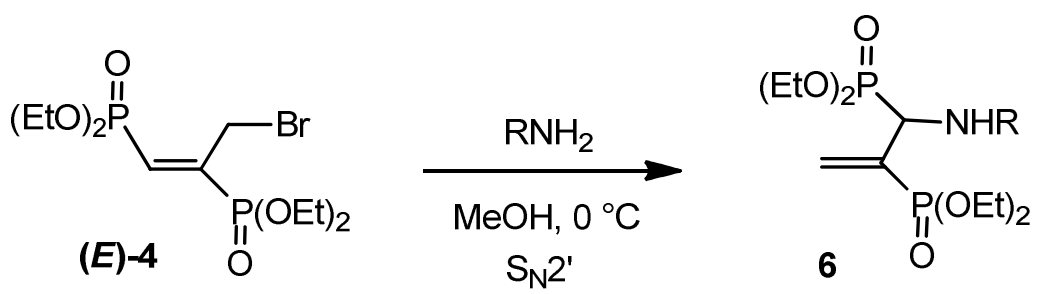

Scheme 5. Synthesis of tetraethyl 1-(alkylamino)prop-2-ene-1,2-diyldiphosphonates 6

Table 2. Synthesis of functionalized allylamines 6a-g

\begin{tabular}{ccccc}
\hline Entry & $\mathrm{R}$ & Time $(\mathrm{h})$ & Product & Yield (\%) \\
\hline 1 & $\mathrm{C}_{6} \mathrm{H}_{5} \mathrm{CH}_{2}$ & 5 & $\mathbf{6 a}$ & 75 \\
2 & $\mathrm{C}_{6} \mathrm{H}_{5} \mathrm{CH}_{2} \mathrm{CH}_{2}$ & 6 & $\mathbf{6 b}$ & 86 \\
3 & $p-\mathrm{MeOC}_{6} \mathrm{H}_{4} \mathrm{CH}_{2}$ & 6 & $\mathbf{6 c}$ & 68 \\
4 & $p-\mathrm{ClC}_{6} \mathrm{H}_{4} \mathrm{CH}_{2}$ & 7 & $\mathbf{6 d}$ & 90 \\
5 & $p-\mathrm{FC}_{6} \mathrm{H}_{4} \mathrm{CH}_{2}$ & 4 & $\mathbf{6 e}$ & 91 \\
6 & $-\mathrm{CH}_{2}$ & 5 & $\mathbf{6 f}$ & 87 \\
7 & & 6 & $\mathbf{6 g}$ & 84 \\
\hline
\end{tabular}

${ }^{*}$ Yields refer to the pure isolated products characterized by ${ }^{1} \mathrm{H},{ }^{13} \mathrm{C}$ NMR.

\section{Conclusions}

We have developed a new strategy for the synthesis of highly stereoselective 2,3difunctionalized allyl bromide $(\boldsymbol{E})-\mathbf{4}$, in addition to the family of allyl bromides we published 
previously, and have shown its direct use in the synthesis of a new family of 1,2diphosphorylated allylamines $\mathbf{6}$ in excellent yields.

\section{Experimental Section}

General. All commercially available chemicals and reagents were used without further purification. All the reactions were monitored by TLC on silica gel plates (Fluka Kieselgel 60 $\mathrm{F}_{254}$ ). Flash column chromatography was performed with $70-230$ mesh silica gel. The ${ }^{1} \mathrm{H}$ and ${ }^{13} \mathrm{C}$ spectra were recorded in $\mathrm{CDCl}_{3}$ at room temperature on a Bruker AMX 300 spectrometer. Some products secured by DEPT 135, NOESY, HMQC and HMBC experiments. Chemical shifts are given in $\delta(\mathrm{ppm})$ and coupling constants $J(\mathrm{~Hz})$ relative to TMS as internal standard. Multiplicities were recorded as s (singlet), d (doublet), dd (doublet of doublets), $t$ (triplet), $d t$ (doublet of triplets), q (quartet), dq (doublet of quartets), br (board) or m (multiplet). Reactions involving anhydrous conditions were conducted in dry glassware under a nitrogen atmosphere. IR spectra were recorded on a Bruker Vertex 70 FT-IR spectrophotometer. High-Resolution Mass Spectrometry (HRMS) analyses were performed in Laberca laboratory at Oniris (NantesAtlantic National College of Veterinary Medicine, FoodScience and Engineering) on a mass spectrometer equipped with a door coupled to a linear Orbitrap (LTQ-Orbitrap of Thermo Fisher Scientific) in positive electrospray ionization.

Diethyl 3-bromoprop-1-en-2-ylphosphonate (1). To a stirred solution of diethyl 1-(hydroxymethyl) vinylphosphonate $(56$ mmoles $)$ in dry ether $(45 \mathrm{~mL})$ was added dropwise a solution of phosphorus tribromide ( 28 mmoles) diluted in $5 \mathrm{~mL}$ of anhydrous ether at $-10{ }^{\circ} \mathrm{C}$ under nitrogen atmosphere. The resulting mixture was left for one hour then hydrolyzed with water in ice bath at $-10{ }^{\circ} \mathrm{C}$ and extracted with hexane $(3 \times 20 \mathrm{~mL})$. The organic layer was washed with brine then dried over $\mathrm{MgSO}_{4}$. After the solvent was evaporated, the crude product was distilled under reduced pressure to produce a colorless oil. Yield: $90 \%$, b.p. $79{ }^{\circ} \mathrm{C} / 0.6 \mathrm{mmHg}$; ${ }^{1} \mathrm{H}-\mathrm{NMR}\left(300 \mathrm{MHz}, \mathrm{CDCl}_{3}\right): 6.26\left(\mathrm{~d}, 1 \mathrm{H},{ }^{3} J_{H P} 22 \mathrm{~Hz},=\mathrm{CH}\right) ; 6.19\left(\mathrm{~d}, 1 \mathrm{H},{ }^{3} J_{H P} 44 \mathrm{~Hz},=\mathrm{CH}\right)$; 4.17-4.07 (m, 6H, 2OCH $\left.2, \mathrm{CH}_{2} \mathrm{Br}\right) ; 1.35\left(\mathrm{t}, 6 \mathrm{H}, J\right.$ 7.5 Hz, $\left.2 \mathrm{CH}_{3}\right) ;{ }^{13} \mathrm{C}-\mathrm{NMR}\left(75 \mathrm{MHz}, \mathrm{CDCl}_{3}\right)$ : $135.8\left(\mathrm{~d},=\mathrm{C},{ }^{1} J_{C P} 177.7 \mathrm{~Hz}\right) ; 134.4\left(\mathrm{~d},=\mathrm{CH}_{2},{ }^{2} J_{C P} 9 \mathrm{~Hz}\right) ; 62.3\left(\mathrm{~d}, 2 \mathrm{OCH}_{2},{ }^{2} J_{C P} 5.2 \mathrm{~Hz}\right) ; 29.7(\mathrm{~d}$, $\left.\mathrm{CH}_{2} \mathrm{Br},{ }^{2} J_{C P} 16.5 \mathrm{~Hz}\right) ; 16.3\left(\mathrm{~d}, 2 \mathrm{CH}_{3},{ }^{3} J_{C P} 6 \mathrm{~Hz}\right) ;{ }^{31} \mathrm{P}-\mathrm{NMR}$ (121 MHz, $\left.\mathrm{CDCl}_{3}\right): 15.01$. $\mathrm{C}_{7} \mathrm{H}_{14} \mathrm{BrO}_{3} \mathrm{P}$ : HRMS calculated for $\left[\mathrm{M}+\mathrm{H}^{+}\right]$256.99367, found 256.99371 .

Representative procedure, tetraethyl prop-2-ene-1,2-diyldiphosphonate (2). A solution of allyl bromide $1(20 \mathrm{mmol})$ and triethylphosphite $(28 \mathrm{mmol})$ was stirred at $80{ }^{\circ} \mathrm{C}$ for 1 hour. When the reaction was completed, the mixture was evaporated under reduced pressure. The oily residue obtained was distilled under reduced pressure to obtain the allylphosphonate 2 . Colorless oil. Yield: $96 \%$, b.p. $130{ }^{\circ} \mathrm{C} / 0.6 \mathrm{mmHg} ;{ }^{1} \mathrm{H}-\mathrm{NMR}\left(300 \mathrm{MHz}, \mathrm{CDCl}_{3}\right): 6.28$ (dd, $1 \mathrm{H},{ }^{3} J_{H P} 48 \mathrm{~Hz}$, $\left.{ }^{4} J_{H P} 6 \mathrm{~Hz},=\mathrm{CH}\right) ; 6.27\left(\mathrm{dd}, 1 \mathrm{H},{ }^{3} J_{H P} 24 \mathrm{~Hz},{ }^{4} J_{H P} 6 \mathrm{~Hz},=\mathrm{CH}\right) ; 4.10(\mathrm{dq}, 8 \mathrm{H}, J 6 \mathrm{~Hz}, J 6 \mathrm{~Hz}$, $\left.4 \mathrm{OCH}_{2}\right) ; 2.77\left(\mathrm{dd}, 2 \mathrm{H},{ }^{2} J_{H P} 21 \mathrm{~Hz},{ }^{3} J_{H P} 12 \mathrm{~Hz}, \mathrm{CH}_{2} \mathrm{P}\right) ; 1.34,1.30(2 \mathrm{t}, 12 \mathrm{H}, J 6 \mathrm{~Hz}, J 6 \mathrm{~Hz}$, 
$\left.4 \mathrm{CH}_{3}\right) ;{ }^{13} \mathrm{C}-\mathrm{NMR}\left(75 \mathrm{MHz}, \mathrm{CDCl}_{3}\right): 133.8\left(\mathrm{t},=\mathrm{CH}_{2},{ }^{2} J_{C P} 16.5 \mathrm{~Hz},{ }^{3} J_{C P} 8.2 \mathrm{~Hz}\right) ; 129.5(\mathrm{dd},=\mathrm{CP}$, $\left.{ }^{1} J_{C P} 180 \mathrm{~Hz},{ }^{2} J_{C P} 30 \mathrm{~Hz}\right) ; 62.1\left(2 \mathrm{~d}, 4 \mathrm{OCH}_{2},{ }^{2} J_{C P} 9 \mathrm{~Hz},{ }^{2} J_{C P} 9 \mathrm{~Hz}\right) ; 27.6\left(\mathrm{dd}, \mathrm{CH}_{2} \mathrm{P},{ }^{1} J_{C P} 140.2\right.$ $\left.\mathrm{Hz},{ }^{2} J_{C P} 12 \mathrm{~Hz}\right) ; 16.3\left(2 \mathrm{~d}, 4 \mathrm{CH}_{3},{ }^{3} J_{C P} 6 \mathrm{~Hz},{ }^{3} J_{C P} 6 \mathrm{~Hz}\right) ;{ }^{31} \mathrm{P}-\mathrm{NMR}\left(121 \mathrm{MHz}, \mathrm{CDCl}_{3}\right): 17.53(\mathrm{~d}$, $=\mathrm{CP}, J 32.6 \mathrm{~Hz}) ; 25.27\left(\mathrm{~d}, \mathrm{CH}_{2} \mathrm{P}, J 32.6 \mathrm{~Hz}\right)$.

Synthesis of $(E)$-tetraethyl 3-bromoprop-1-ene-1,2-diyldiphosphonate $(E)$-4 and tetraethyl 3-bromoprop-2-ene-1,2-diyldiphosphonate $(\boldsymbol{E})$-5. A solution of bromine $(74 \mathrm{mmol})$ in carbon tetrachloride $(28 \mathrm{~mL})$ was added dropwise to a refluxing solution of allylphosphonate 2 (71 mmol) in carbon tetrachloride $(160 \mathrm{~mL})$ at such a rate that the bromine color gradually disappeared. The end of the reaction is indicated by the persistence of a brownish color. Excess of bromine was removed by washing with aqueous solution of sodium thiosulfate. The organic layer was then washed with brine and dried over magnesium sulfate. Filtration and removal of the solvent gave a residue 3 . DBU $(12 \mathrm{mmol})$ was added to a solution of 3 (11 mmol) in acetonitrile $(60 \mathrm{~mL})$. The mixture was stirred for $48 \mathrm{~h}$ at room temperature. After evaporation of the solvent, the residue was separated by chromatography on silica gel $\left(\mathrm{CH}_{2} \mathrm{Cl}_{2}-\mathrm{MeOH}, 97: 3\right)$ affording pure compounds $\mathbf{4}$ and $\mathbf{5}$.

Tetraethyl (E)-3-bromoprop-1-ene-1,2-diyldiphosphonate (4). Yellow liquid. Yield: 73\%, IR (neat): $1643,1240 \mathrm{~cm}^{-1}$. ${ }^{1} \mathrm{H}-\mathrm{NMR}\left(300 \mathrm{MHz}, \mathrm{CDCl}_{3}\right): 6.70\left(\mathrm{dd}, 1 \mathrm{H},{ }^{2} J_{H P} 27 \mathrm{~Hz},{ }^{3} J_{H P} 18 \mathrm{~Hz}\right.$, $=\mathrm{CH}) ; 4.60\left(\mathrm{~d}, 2 \mathrm{H},{ }^{3} J_{H P} 18 \mathrm{~Hz}, \mathrm{CH}_{2} \mathrm{Br}\right) ; 4.22-4.13\left(\mathrm{~m}, 8 \mathrm{H}, 4 \mathrm{OCH}_{2}\right) ; 1.38,1.36,(2 \mathrm{t}, 6 \mathrm{H}, J 6 \mathrm{~Hz}, J$ $\left.6 \mathrm{~Hz}, 4 \mathrm{CH}_{3}\right) ;{ }^{13} \mathrm{C}-\mathrm{NMR}\left(75 \mathrm{MHz}, \mathrm{CDCl}_{3}\right): 146.6\left(\mathrm{dd},=\mathrm{C},{ }^{1} J_{C P} 169.5 \mathrm{~Hz},{ }^{2} J_{C P} 5.2 \mathrm{~Hz}\right) ; 133.6(\mathrm{dd}$, $\left.=\mathrm{CH},{ }^{1} J_{C P} 171 \mathrm{~Hz},{ }^{2} J_{C P} 10.5 \mathrm{~Hz}\right) ; 62.6,62.2,\left(2 \mathrm{~d}, 4 \mathrm{OCH}_{2},{ }^{2} J_{C P} 6 \mathrm{~Hz},{ }^{2} J_{C P} 6 \mathrm{~Hz}\right) ; 23.2\left(\mathrm{t}, \mathrm{CH}_{2} \mathrm{Br}\right.$, $\left.{ }^{2} J_{C P}{ }^{3} J_{C P} 9 \mathrm{~Hz}\right) ; 16.0\left(\mathrm{~d}, 2 \mathrm{CH}_{3},{ }^{3} J_{C P} 6 \mathrm{~Hz}\right) ; 15.9\left(\mathrm{~d}, 2 \mathrm{CH}_{3},{ }^{3} J_{C P} 6.7 \mathrm{~Hz}\right) ;{ }^{31} \mathrm{P}-\mathrm{NMR}(121 \mathrm{MHz}$, $\left.\mathrm{CDCl}_{3}\right): 13.95(\mathrm{~d},=\mathrm{CP}, J 91.9 \mathrm{~Hz}) ; 11.02\left(\mathrm{~d}, \mathrm{CH}_{2} \mathrm{P}, J 91.9 \mathrm{~Hz}\right) . \mathrm{C}_{11} \mathrm{H}_{23} \mathrm{BrO}_{6} \mathrm{P}_{2}: \mathrm{HRMS}$ calculated for $\left[\mathrm{M}+\mathrm{H}^{+}\right]$393.02260, found 393.02219.

Tetraethyl (E)-3-bromoprop-2-ene-1,2-diyldiphosphonate (5). Yellow liquid. Yield: $8 \%,{ }^{1} \mathrm{H}-$ NMR $\left(300 \mathrm{MHz}, \mathrm{CDCl}_{3}\right): 7.48\left(\mathrm{dd}, 1 \mathrm{H},{ }^{3} J_{H P} 14.4 \mathrm{~Hz},{ }^{4} J_{H P} 6 \mathrm{~Hz},=\mathrm{CH}\right) ; 4.16-4.08(\mathrm{~m}, 8 \mathrm{H}$, $\left.4 \mathrm{OCH}_{2}\right) ; 3.04\left(\mathrm{dd}, 2 \mathrm{H},{ }^{2} J_{H P} 24 \mathrm{~Hz},{ }^{3} J_{H P} 18 \mathrm{~Hz}, \mathrm{CH}_{2} \mathrm{P}\right) ; 1.38,1.32$, (2t, $6 \mathrm{H}, J 6 \mathrm{~Hz}, J 6 \mathrm{~Hz}$, $\left.4 \mathrm{CH}_{3}\right) ;{ }^{13} \mathrm{C}-\mathrm{NMR}\left(75 \mathrm{MHz}, \mathrm{CDCl}_{3}\right.$ ): 128.6 (dd, =C, ${ }^{1} J_{C P} 174.7 \mathrm{~Hz},{ }^{2} J_{C P} 11.2 \mathrm{~Hz}$ ); 127.0 (dd, $\left.=\mathrm{CH},{ }^{2} J_{C P} 22.8 \mathrm{~Hz},{ }^{3} J_{C P} 12.7 \mathrm{~Hz}\right) ; 62.9,62.7,\left(2 \mathrm{~d}, 4 \mathrm{OCH}_{2},{ }^{2} J_{C P} 6 \mathrm{~Hz},{ }^{2} J_{C P} 6 \mathrm{~Hz}\right) ; 29.4\left(\mathrm{dd}, \mathrm{CH}_{2} \mathrm{P}\right.$, $\left.{ }^{1} J_{C P} 140.2 \mathrm{~Hz},{ }^{2} J_{C P} 9 \mathrm{~Hz}\right) ; 16.4\left(\mathrm{~d}, 2 \mathrm{CH}_{3},{ }^{3} J_{C P} 6 \mathrm{~Hz}\right) ; 16.2\left(\mathrm{~d}, 2 \mathrm{CH}_{3},{ }^{3} J_{C P} 6.7 \mathrm{~Hz}\right) ;{ }^{31} \mathrm{P}-\mathrm{NMR}(121$ $\left.\mathrm{MHz}, \mathrm{CDCl}_{3}\right)$ : 13.64 (d, =CP, J 8.4 Hz); 22.29 (d, $\left.\mathrm{CH}_{2} \mathrm{P}, J 8.4 \mathrm{~Hz}\right)$.

Synthesis model of tetraethyl 3-(alkylamino)prop-1-ene-1,2-diyldiphosphonate 6 (a-g). To a solution of allyl bromide $(\boldsymbol{E})-4(0.2 \mathrm{~g}, 0.5 \mathrm{mmol})$ diluted in $3 \mathrm{~mL}$ of absolute methanol was added dropwise primary amine $(1 \mathrm{mmol})$ at $0^{\circ} \mathrm{C}$. After stirring during the time indicated in table $(\mathbf{1})$, the mixture was concentrated and the organic residue obtained was purified by chromatography on silica gel $\left(\mathrm{CH}_{2} \mathrm{Cl}_{2}-\mathrm{MeOH}, 9.6: 0.4\right)$.

Tetraethyl 1-(benzylamino)prop-2-ene-1,2-diyldiphosphonate (6a). Yellow liquid. Yield: $75 \%$, IR (neat): 3392, 1649, $1246 \mathrm{~cm}^{-1} .{ }^{1} \mathrm{H}-\mathrm{NMR}\left(300 \mathrm{MHz}, \mathrm{CDCl}_{3}\right): 7.29$ (m, 5H, aromatic H); $6.46\left(\mathrm{t}, 1 \mathrm{H},{ }^{2} J_{H H}{ }^{3} J_{H P} 3 \mathrm{~Hz},=\mathrm{CH}\right) ; 6.35\left(\mathrm{dd}, 1 \mathrm{H},{ }^{2} J_{H H} 3 \mathrm{~Hz},{ }^{3} J_{H P} 18 \mathrm{~Hz},=\mathrm{CH}\right) ; 4.25-4.08(\mathrm{~m}, 8 \mathrm{H}$, $\left.4 \mathrm{OCH}_{2}\right) ; 3.87\left(\mathrm{dd}, 1 \mathrm{H},{ }^{2} J_{H P} 21 \mathrm{~Hz},{ }^{3} J_{H P} 15 \mathrm{~Hz}, \mathrm{CHP}\right) ; 3.91-3.72\left(\mathrm{AB}, 2 \mathrm{H}, J 12 \mathrm{~Hz}, \mathrm{CH}_{2} \mathrm{~N}\right) ; 2.84$ (br, s, 1H, NH); 1.34, 1.32, $1.30\left(3 \mathrm{t}, 12 \mathrm{H}, J 6 \mathrm{~Hz}, J 6 \mathrm{~Hz}, J 6 \mathrm{~Hz}, 4 \mathrm{CH}_{3}\right) ;{ }^{13} \mathrm{C}-\mathrm{NMR}(75 \mathrm{MHz}$, 
$\left.\mathrm{CDCl}_{3}\right): 139.4$ ( aromatic C); $136.3\left(\mathrm{~d},=\mathrm{C},{ }^{1} J_{C P} 174.7 \mathrm{~Hz}\right) ; 134.0\left(\mathrm{t},=\mathrm{CH}_{2},{ }^{2} J_{C P} 8.2 \mathrm{~Hz},{ }^{3} J_{C P} 7.5\right.$ $\mathrm{Hz}$ ); 129.0, 128.3, 128.3, 127.1, (aromatic C); 63.3, $62.7\left(2 \mathrm{~d}, 2 \mathrm{OCH}_{2},{ }^{2} J_{C P} 7.5 \mathrm{~Hz},{ }^{2} J_{C P} 7.5 \mathrm{~Hz}\right.$ ); 62.3, $62.1\left(2 \mathrm{~d}, 2 \mathrm{OCH}_{2},{ }^{2} J_{C P} 6 \mathrm{~Hz},{ }^{2} J_{C P} 6 \mathrm{~Hz}\right) ; 55.1\left(\mathrm{dd}, \mathrm{CHP},{ }^{1} J_{C P} 156.7 \mathrm{~Hz},{ }^{2} J_{C P} 12.7 \mathrm{~Hz}\right) ; 51.5$ $\left(\mathrm{d}, \mathrm{CH}_{2} \mathrm{~N},{ }^{3} J_{C P} 15 \mathrm{~Hz}\right) ; 16.5,16.4,\left(2 \mathrm{~d}, 4 \mathrm{CH}_{3},{ }^{3} J_{C P} 6 \mathrm{~Hz},{ }^{3} J_{C P} 6 \mathrm{~Hz}\right) ;{ }^{31} \mathrm{P}-\mathrm{NMR}(121 \mathrm{MHz}$, $\mathrm{CDCl}_{3}$ ): 16.92 (d, =CP, $\left.J 26.6 \mathrm{~Hz}\right) ; 22.19$ (d, CHP, $\left.J 26.6 \mathrm{~Hz}\right) . \mathrm{C}_{18} \mathrm{H}_{31} \mathrm{NO}_{6} \mathrm{P}_{2}$ : HRMS calculated for $\left[\mathrm{M}+\mathrm{H}^{+}\right]$420.16994, found 420.16956 .

Tetraethyl 1-(phenethylamino)prop-2-ene-1,2-diyldiphosphonate (6b). Green liquid. Yield: $86 \%$, IR (neat): $3421,1651,1233 \mathrm{~cm}^{-1} .{ }^{1} \mathrm{H}-\mathrm{NMR}\left(300 \mathrm{MHz}, \mathrm{CDCl}_{3}\right): 7.23$ (m, 5H, aromatic $\mathrm{H}$ ); $6.35\left(\mathrm{dd}, 1 \mathrm{H},{ }^{2} J_{H H} 3 \mathrm{~Hz},{ }^{3} J_{H P} 15 \mathrm{~Hz},=\mathrm{CH}\right) ; 6.23\left(\mathrm{dd}, 1 \mathrm{H},{ }^{2} J_{H H} 3 \mathrm{~Hz},{ }^{3} J_{H P} 39 \mathrm{~Hz},=\mathrm{CH}\right) ; 4.16-$ $4.03\left(\mathrm{~m}, 8 \mathrm{H}, 4 \mathrm{OCH}_{2}\right) ; 3.83\left(\mathrm{dd}, 1 \mathrm{H},{ }^{2} J_{H P} 21 \mathrm{~Hz},{ }^{3} J_{H P} 12 \mathrm{~Hz}, \mathrm{CHP}\right) ; 2.99-2.72\left(\mathrm{~m}, 4 \mathrm{H}, 2 \mathrm{CH}_{2}\right)$; 1.99 (br, s, 1H, NH); 1.34, 1.32, (2t, $\left.12 \mathrm{H}, J 6 \mathrm{~Hz}, J 6 \mathrm{~Hz}, 4 \mathrm{CH}_{3}\right) ;{ }^{13} \mathrm{C}-\mathrm{NMR}\left(75 \mathrm{MHz}, \mathrm{CDCl}_{3}\right)$ : 139.8 (aromatic C); $136.3\left(\mathrm{~d},=\mathrm{C},{ }^{1} J_{C P} 174.7 \mathrm{~Hz}\right) ; 133.6\left(\mathrm{t},=\mathrm{CH}_{2},{ }^{2} J_{C P} 8.2 \mathrm{~Hz},{ }^{3} J_{C P} 7.5 \mathrm{~Hz}\right)$; 128.7, 128.3, 126.1, (aromatic C); 63.2, $62.6\left(2 \mathrm{~d}, 2 \mathrm{OCH}_{2},{ }^{2} J_{C P} 7.5 \mathrm{~Hz},{ }^{2} J_{C P} 7.5 \mathrm{~Hz}\right) ; 62.2,62.0$ $\left(2 \mathrm{~d}, 2 \mathrm{OCH}_{2},{ }^{2} J_{C P} 6 \mathrm{~Hz},{ }^{2} J_{C P} 6 \mathrm{~Hz}\right) ; 55.4\left(\mathrm{dd}, \mathrm{CHP},{ }^{1} J_{C P} 156.7 \mathrm{~Hz},{ }^{2} J_{C P} 12.7 \mathrm{~Hz}\right) ; 48.9\left(\mathrm{~d}, \mathrm{CH}_{2} \mathrm{~N}\right.$, $\left.{ }^{3} J_{C P} 13.5 \mathrm{~Hz}\right) ; 36.3\left(\mathrm{~s}, \mathrm{CH}_{2}\right) ; 16.4\left(\mathrm{~d}, 2 \mathrm{CH}_{3},{ }^{3} J_{C P} 5.2 \mathrm{~Hz}\right) ; 16.3\left(\mathrm{~d}, 2 \mathrm{CH}_{3},{ }^{3} J_{C P} 6 \mathrm{~Hz}\right) ;{ }^{31} \mathrm{P}-\mathrm{NMR}$ $\left(121 \mathrm{MHz}, \mathrm{CDCl}_{3}\right): 17.16$ (d, =CP, J 29.0 Hz); 22.31 (d, CHP, J $\left.29.0 \mathrm{~Hz}\right) . \mathrm{C}_{19} \mathrm{H}_{33} \mathrm{NO}_{6} \mathrm{P}_{2}$ : HRMS calculated for $\left[\mathrm{M}+\mathrm{H}^{+}\right] 434.18559$, found 434.18504 .

Tetraethyl 1-(4-methoxybenzylamino)prop-2-ene-1,2-diyldiphosphonate (6c). Yellow liquid. Yield: $68 \%$, IR (neat): 3368, 1642, $1238 \mathrm{~cm}^{-1} .{ }^{1} \mathrm{H}-\mathrm{NMR}\left(300 \mathrm{MHz}, \mathrm{CDCl}_{3}\right): 7.24$ (d, 2H, $J_{H H} 9$ $\mathrm{Hz}$, aromatic $\mathrm{H}) ; 6.83\left(\mathrm{~d}, 2 \mathrm{H}, J_{H H} 9 \mathrm{~Hz}\right.$, aromatic $\left.\mathrm{H}\right) ; 6.44\left(\mathrm{t}, 1 \mathrm{H},{ }^{2} J_{H H}{ }^{3} J_{H P} 3 \mathrm{~Hz},=\mathrm{CH}\right) ; 6.33(\mathrm{dd}$, $\left.1 \mathrm{H},{ }^{2} J_{H H} 3 \mathrm{~Hz},{ }^{3} J_{H P} 21 \mathrm{~Hz},=\mathrm{CH}\right) ; 4.21-4.08\left(\mathrm{~m}, 8 \mathrm{H}, 4 \mathrm{OCH}_{2}\right) ; 3.85\left(\mathrm{dd}, 1 \mathrm{H},{ }^{2} J_{H P} 21 \mathrm{~Hz},{ }^{3} J_{H P} 15\right.$ $\mathrm{Hz}, \mathrm{CHP}) ; 3.79$ (s, 3H, $\left.\mathrm{CH}_{3}\right) ; 3.83-3.65\left(\mathrm{AB}, 2 \mathrm{H}, J 12 \mathrm{~Hz}, \mathrm{CH}_{2} \mathrm{~N}\right) ; 2.58$ (br, s, $\left.1 \mathrm{H}, \mathrm{NH}\right) ; 1.34$, 1.28, (2t, $\left.12 \mathrm{H}, J 6 \mathrm{~Hz}, J 6 \mathrm{~Hz}, 4 \mathrm{CH}_{3}\right) ;{ }^{13} \mathrm{C}-\mathrm{NMR}\left(75 \mathrm{MHz}, \mathrm{CDCl}_{3}\right): 158.7$ (aromatic C); 136.3 (d, $\left.=\mathrm{C},{ }^{1} J_{C P} 175.7 \mathrm{~Hz}\right) ; 133.9\left(\mathrm{t},=\mathrm{CH}_{2},{ }^{2} J_{C P} 8.2 \mathrm{~Hz},{ }^{3} J_{C P} 7.5 \mathrm{~Hz}\right) ; 131.5,129.4$, 113.6, (aromatic C); $63.1,62.5\left(2 \mathrm{~d}, 2 \mathrm{OCH}_{2},{ }^{2} J_{C P} 6.7 \mathrm{~Hz},{ }^{2} J_{C P} 6.7 \mathrm{~Hz}\right) ; 62.1,62.0\left(2 \mathrm{~d}, 2 \mathrm{OCH}_{2},{ }^{2} J_{C P} 6 \mathrm{~Hz},{ }^{2} J_{C P} 6 \mathrm{~Hz}\right)$; $54.9\left(\mathrm{dd}, \mathrm{CHP},{ }^{1} J_{C P} 156.7 \mathrm{~Hz},{ }^{2} J_{C P} 12.7 \mathrm{~Hz}\right) ; 55.1\left(\mathrm{OCH}_{3}\right) ; 50.8\left(\mathrm{~d}, \mathrm{CH}_{2} \mathrm{~N},{ }^{3} J_{C P} 15 \mathrm{~Hz}\right) ; 16.4$, $16.2\left(2 \mathrm{~d}, 4 \mathrm{CH}_{3},{ }^{3} J_{C P} 6 \mathrm{~Hz},{ }^{3} J_{C P} 6 \mathrm{~Hz}\right) ;{ }^{31} \mathrm{P}-\mathrm{NMR}\left(121 \mathrm{MHz}, \mathrm{CDCl}_{3}\right): 16.95$ (d, =CP, J $25.4 \mathrm{~Hz}$ ); 22.17 (d, CHP, $J 25.4 \mathrm{~Hz}$ ) $\mathrm{C}_{19} \mathrm{H}_{33} \mathrm{NO}_{7} \mathrm{P}_{2}$ : HRMS calculated for $\left[\mathrm{M}+\mathrm{H}^{+}\right]$450.18050, found 450.17976 .

Tetraethyl 1-(4-chlorobenzylamino)prop-2-ene-1,2-diyldiphosphonate (6d). Yellow liquid. Yield: $90 \%$, IR (neat): 3336, 1646, $1260 \mathrm{~cm}^{-1} .{ }^{1} \mathrm{H}-\mathrm{NMR}\left(300 \mathrm{MHz}, \mathrm{CDCl}_{3}\right): 7.28(\mathrm{~m}, 4 \mathrm{H}$, aromatic $\mathrm{H}) ; 6.44\left(\mathrm{~d}, 1 \mathrm{H},{ }^{3} J_{H P} 3 \mathrm{~Hz},=\mathrm{CH}\right) ; 6.33\left(\mathrm{dd}, 1 \mathrm{H},{ }^{2} J_{H H} 3 \mathrm{~Hz},{ }^{3} J_{H P} 21 \mathrm{~Hz},=\mathrm{CH}\right) ; 4.21-4.08$ $\left(\mathrm{m}, 8 \mathrm{H}, 4 \mathrm{OCH}_{2}\right) ; 3.82\left(\mathrm{dd}, 1 \mathrm{H},{ }^{2} J_{H P} 21 \mathrm{~Hz},{ }^{3} J_{H P} 15 \mathrm{~Hz}, \mathrm{CHP}\right) ; 3.87-3.69(\mathrm{AB}, 2 \mathrm{H}, J 12 \mathrm{~Hz}$, $\left.\mathrm{CH}_{2} \mathrm{~N}\right) ; 2.26$ (br, s, $\left.1 \mathrm{H}, \mathrm{NH}\right) ; 1.34,1.32,1.28\left(3 \mathrm{t}, 12 \mathrm{H}, J 6 \mathrm{~Hz}, J 6 \mathrm{~Hz}, J 6 \mathrm{~Hz}, 4 \mathrm{CH}_{3}\right) ;{ }^{13} \mathrm{C}-\mathrm{NMR}$ $\left(75 \mathrm{MHz}, \mathrm{CDCl}_{3}\right): 138.0$ (aromatic C); $136.3\left(\mathrm{~d},=\mathrm{C},{ }^{1} J_{C P} 173.2 \mathrm{~Hz}\right) ; 133.9\left(\mathrm{t},=\mathrm{CH}_{2},{ }^{2} J_{C P} 8.2 \mathrm{~Hz}\right.$, $\left.{ }^{3} J_{C P} 7.5 \mathrm{~Hz}\right) ; 132.8,129.6,128.4$, (aromatic C); 63.2, $62.7\left(2 \mathrm{~d}, 2 \mathrm{OCH}_{2},{ }^{2} J_{C P} 7.5 \mathrm{~Hz},{ }^{2} J_{C P} 7.5 \mathrm{~Hz}\right.$ ); 62.3, $62.1\left(2 \mathrm{~d}, 2 \mathrm{OCH}_{2},{ }^{2} J_{C P} 6 \mathrm{~Hz},{ }^{2} J_{C P} 6 \mathrm{~Hz}\right) ; 55.0\left(\mathrm{dd}, \mathrm{CHP},{ }^{1} J_{C P} 157.5 \mathrm{~Hz},{ }^{2} J_{C P} 13.5 \mathrm{~Hz}\right) ; 50.6$ $\left(\mathrm{d}, \mathrm{CH}_{2} \mathrm{~N},{ }^{3} J_{C P} 15 \mathrm{~Hz}\right) ; 16.5,16.3\left(2 \mathrm{~d}, 4 \mathrm{CH}_{3},{ }^{3} J_{C P} 6.7 \mathrm{~Hz},{ }^{3} J_{C P} 6.7 \mathrm{~Hz}\right) ;{ }^{31} \mathrm{P}-\mathrm{NMR}(121 \mathrm{MHz}$, $\mathrm{CDCl}_{3}$ ): $16.80\left(\mathrm{~d},=\mathrm{CP}, J 25.4 \mathrm{z}\right.$ ); 22.17 (d, CHP, $J 25.4 \mathrm{~Hz}$ ). $\mathrm{C}_{18} \mathrm{H}_{30} \mathrm{ClNO}_{6} \mathrm{P}_{2}$ : HRMS calculated for $\left[\mathrm{M}+\mathrm{H}^{+}\right]$454.13096, found 454.13043. 
Tetraethyl 1-(4-fluorobenzylamino)prop-2-ene-1,2-diyldiphosphonate (6e). Orange liquid. Yield: 91\%, IR (neat): 3389, 1647, $1245 \mathrm{~cm}^{-1} .{ }^{1} \mathrm{H}-\mathrm{NMR}$ (300 MHz, $\mathrm{CDCl}_{3}$ ): 7.3 (dd, $2 \mathrm{H},{ }^{3} J_{H F} 9$ $\mathrm{Hz},{ }^{3} J_{H P} 6 \mathrm{~Hz}$, aromatic $\left.\mathrm{H}\right) ; 6.98\left(\mathrm{t}, 2 \mathrm{H},{ }^{3} J_{H H}{ }^{4} J_{H F} 9 \mathrm{~Hz}\right.$, aromatic $\left.\mathrm{H}\right) ; 6.45\left(\mathrm{t}, 1 \mathrm{H},{ }^{2} J_{H H}{ }^{3} J_{H P} 3 \mathrm{~Hz}\right.$, $=\mathrm{CH}) ; 6.33\left(\mathrm{dd}, 1 \mathrm{H},{ }^{2} J_{H H} 3 \mathrm{~Hz},{ }^{3} J_{H P} 21 \mathrm{~Hz},=\mathrm{CH}\right) ; 4.19-4.08\left(\mathrm{~m}, 8 \mathrm{H}, 4 \mathrm{OCH}_{2}\right) ; 3.83\left(\mathrm{dd}, 1 \mathrm{H},{ }^{2} J_{H P}\right.$ $\left.21 \mathrm{~Hz},{ }^{3} J_{H P} 12 \mathrm{~Hz}, \mathrm{CHP}\right) ; 3,87-3.69\left(\mathrm{AB}, 2 \mathrm{H}, J 12 \mathrm{~Hz}, \mathrm{CH}_{2} \mathrm{~N}\right) ; 2.53$ (br, s, 1H, NH); 1.34, 1.30 $\left(2 \mathrm{t}, 12 \mathrm{H}, J 6 \mathrm{~Hz}, J 6 \mathrm{~Hz}, 4 \mathrm{CH}_{3}\right) ;{ }^{13} \mathrm{C}-\mathrm{NMR}\left(75 \mathrm{MHz}, \mathrm{CDCl}_{3}\right): 161.9$ (d, aromatic C, ${ }^{1} J_{C F} 243.7$ $\mathrm{Hz}) ; 137.5$ (aromatic C); $134.4\left(\mathrm{dd},=\mathrm{C},{ }^{1} J_{C P} 111 \mathrm{~Hz},{ }^{2} J_{C P} 8.2 \mathrm{~Hz}\right) ; 133.8\left(\mathrm{t},=\mathrm{CH}_{2},{ }^{2} J_{C P} 8.2 \mathrm{~Hz}\right.$, ${ }^{3} J_{C P} 7.5 \mathrm{~Hz}$ ); 129.8, 129.7, 115.1, 114.88, (aromatic C); 63.1, $62.6\left(2 \mathrm{~d}, 2 \mathrm{OCH}_{2},{ }^{2} J_{C P} 6.7 \mathrm{~Hz},{ }^{2} J_{C P}\right.$ $6.7 \mathrm{~Hz}) ; 62.2,62.0\left(2 \mathrm{~d}, 2 \mathrm{OCH}_{2},{ }^{2} J_{C P} 6 \mathrm{~Hz},{ }^{2} J_{C P} 6 \mathrm{~Hz}\right) ; 54.9\left(\mathrm{dd}, \mathrm{CHP},{ }^{1} J_{C P} 156.7 \mathrm{~Hz},{ }^{2} J_{C P} 12.7\right.$ $\mathrm{Hz}) ; 50.6\left(\mathrm{~d}, \mathrm{CH}_{2} \mathrm{~N},{ }^{3} J_{C P} 15 \mathrm{~Hz}\right) ; 16.4,16.2,\left(2 \mathrm{~d}, 4 \mathrm{CH}_{3},{ }^{3} J_{C P} 6 \mathrm{~Hz},{ }^{3} J_{C P} 6 \mathrm{~Hz}\right) ;{ }^{31} \mathrm{P}-\mathrm{NMR}(121$ $\mathrm{MHz}, \mathrm{CDCl}_{3}$ ): 16.86 (d, =CP, J $25.4 \mathrm{~Hz}$ ); 22.22 (d, CHP, J $25.4 \mathrm{~Hz}$ ); ${ }^{19} \mathrm{~F}-\mathrm{NMR}(282 \mathrm{MHz}$, $\mathrm{CDCl}_{3}$ ): $-115.85 . \mathrm{C}_{18} \mathrm{H}_{30} \mathrm{FNO}_{6} \mathrm{P}_{2}$ : HRMS calculated for $\left[\mathrm{M}+\mathrm{H}^{+}\right]$438.16052, found 438.16003.

Tetraethyl 1-(furfurylamino)prop-2-ene-1,2-diyldiphosphonate (6f). Yellow liquid. Yield: 87\%, IR (neat): 3417, 1644, $1250 \mathrm{~cm}^{-1} .{ }^{1} \mathrm{H}-\mathrm{NMR}$ (300 MHz, $\mathrm{CDCl}_{3}$ ): 7.34 (m, 1H, =CH); 6.46 $\left(\mathrm{dd}, 1 \mathrm{H},{ }^{2} J_{H H} 3 \mathrm{~Hz},{ }^{3} J_{H P} 9 \mathrm{~Hz},=\mathrm{CH}\right) ; 6.34\left(\mathrm{dd}, 1 \mathrm{H},{ }^{2} J_{H H} 3 \mathrm{~Hz},{ }^{3} J_{H P} 12 \mathrm{~Hz},=\mathrm{CH}\right) ; 6.29(\mathrm{t}, 1 \mathrm{H}, J 3$ $\mathrm{Hz},=\mathrm{CH}) ; 6.21(\mathrm{~d}, 1 \mathrm{H}, J 3 \mathrm{~Hz},=\mathrm{CH}) ; 4.23-4.08\left(\mathrm{~m}, 8 \mathrm{H}, 4 \mathrm{OCH}_{2}\right) ; 3.87\left(\mathrm{dd}, 1 \mathrm{H},{ }^{2} J_{H P} 21 \mathrm{~Hz},{ }^{3} J_{H P}\right.$ $12 \mathrm{~Hz}, \mathrm{CHP}$ ); 3.88-3.72 (AB, 2H, J $12 \mathrm{~Hz}, \mathrm{CH}_{2} \mathrm{~N}$ ); 2.35 (br, s, 1H, NH); 1.34, 1.28 (2t, $12 \mathrm{H}, J 6$ $\left.\mathrm{Hz}, J 6 \mathrm{~Hz}, 4 \mathrm{CH}_{3}\right) ;{ }^{13} \mathrm{C}-\mathrm{NMR}\left(75 \mathrm{MHz}, \mathrm{CDCl}_{3}\right): 153.0,141.8,(=\mathrm{C}) ; 136.0$ (d, =C, ${ }^{1} J_{C P} 175.5$ $\mathrm{Hz}) ; 134.2\left(\mathrm{t},=\mathrm{CH}_{2},{ }^{2} J_{C P} 8.2 \mathrm{~Hz},{ }^{3} J_{C P} 7.5 \mathrm{~Hz}\right) ; 110.1,107.4,(=\mathrm{C}) ; 63.3,62.8\left(2 \mathrm{~d}, 2 \mathrm{OCH}_{2},{ }^{2} J_{C P}\right.$ $\left.6.7 \mathrm{~Hz},{ }^{2} J_{C P} 6.7 \mathrm{~Hz}\right) ; 62.3,62.2\left(2 \mathrm{~d}, 2 \mathrm{OCH}_{2},{ }^{2} J_{C P} 6 \mathrm{~Hz},{ }^{2} J_{C P} 6 \mathrm{~Hz}\right) ; 54.4\left(\mathrm{dd}, \mathrm{CHP},{ }^{1} J_{C P} 156.7 \mathrm{~Hz}\right.$, $\left.{ }^{2} J_{C P} 13.5 \mathrm{~Hz}\right) ; 44.0\left(\mathrm{~d}, \mathrm{CH}_{2} \mathrm{~N},{ }^{3} J_{C P} 15 \mathrm{~Hz}\right) ; 16.4,16.3\left(2 \mathrm{~d}, 4 \mathrm{CH}_{3},{ }^{3} J_{C P} 6 \mathrm{~Hz},{ }^{3} J_{C P} 6 \mathrm{~Hz}\right) ;{ }^{31} \mathrm{P}-\mathrm{NMR}$ $\left(121 \mathrm{MHz}, \mathrm{CDCl}_{3}\right.$ ): 22.05 (d, =CP, J $29.0 \mathrm{~Hz}$ ); 17 (d, CHP, J 29.0 Hz). $\mathrm{C}_{16} \mathrm{H}_{29} \mathrm{NO}_{7} \mathrm{P}_{2}$ : HRMS calculated for $\left[\mathrm{M}+\mathrm{H}^{+}\right] 410.14920$, found 410.14865 .

Tetraethyl 1-(2-picolylamino)prop-2-ene-1,2-diyldiphosphonate (6g). Yellow liquid. Yield: 84\%, IR (neat): 3422, 1646, $1230 \mathrm{~cm}^{-1} .{ }^{1} \mathrm{H}-\mathrm{NMR}\left(300 \mathrm{MHz}, \mathrm{CDCl}_{3}\right.$ ): 8.52 (dd, 1H, J 4.8 Hz, J $1.8 \mathrm{~Hz},=\mathrm{CHN}) ; 7.64(\mathrm{td}, 1 \mathrm{H}, J 1.8 \mathrm{~Hz}, J 7.8 \mathrm{~Hz},=\mathrm{CH}) ; 7.38(\mathrm{~d}, 1 \mathrm{H}, J 7.8 \mathrm{~Hz},=\mathrm{CH}) ; 7.16(\mathrm{dd}$, $1 \mathrm{H}, J 4.8 \mathrm{~Hz}, J 7 \mathrm{~Hz},=\mathrm{CH}) ; 6.44\left(\mathrm{dd}, 1 \mathrm{H},{ }^{2} J_{H H} 4.2 \mathrm{~Hz},{ }^{3} J_{H P} 20.4 \mathrm{~Hz},=\mathrm{CH}\right) ; 6.37(\mathrm{t}, 1 \mathrm{H}$, $\left.{ }^{2} J_{H H}{ }^{3} J_{H P} 3 \mathrm{~Hz},=\mathrm{CH}\right) ; 4.27-4.07\left(\mathrm{~m}, 8 \mathrm{H}, 4 \mathrm{OCH}_{2}\right) ; 4.03-3.87\left(\mathrm{AB}, 2 \mathrm{H}, J 12 \mathrm{~Hz}, \mathrm{CH}_{2} \mathrm{~N}\right) ; 3.90(\mathrm{dd}$, $\left.1 \mathrm{H},{ }^{2} J_{H P} 21 \mathrm{~Hz},{ }^{3} J_{H P} 12 \mathrm{~Hz}, \mathrm{CHP}\right) ; 2.49$ (br, s, 1H, NH); 1.36, 1.32, (2t, 12H, J $6 \mathrm{~Hz}, J 6 \mathrm{~Hz}$, $\left.4 \mathrm{CH}_{3}\right) ;{ }^{13} \mathrm{C}-\mathrm{NMR}\left(75 \mathrm{MHz}, \mathrm{CDCl}_{3}\right): 159.1$ (=C); $149.0(=\mathrm{C}) ; 136.5(=\mathrm{C}) ; 135.8$ (d, =CP, $\left.{ }^{1} J_{C P} 175.5 \mathrm{~Hz}\right) ; 134.1\left(\mathrm{t},=\mathrm{CH}_{2},{ }^{2} J_{C P} 8.2 \mathrm{~Hz},{ }^{3} J_{C P} 7.5 \mathrm{~Hz}\right) ; 122.2,122.0,(=\mathrm{C}) ; 63.2,62.8(2 \mathrm{~d}$, $\left.2 \mathrm{OCH}_{2},{ }^{2} J_{C P} 7.5 \mathrm{~Hz},{ }^{2} J_{C P} 7.5 \mathrm{~Hz}\right) ; 62.3,62.2\left(2 \mathrm{~d}, 2 \mathrm{OCH}_{2},{ }^{2} J_{C P} 6.7 \mathrm{~Hz},{ }^{2} J_{C P} 6.7 \mathrm{~Hz}\right) ; 55.3(\mathrm{dd}$, $\left.\mathrm{CHP},{ }^{1} J_{C P} 156.7 \mathrm{~Hz},{ }^{2} J_{C P} 13.5 \mathrm{~Hz}\right) ; 52.7\left(\mathrm{~d}, \mathrm{CH}_{2} \mathrm{~N},{ }^{3} J_{C P} 14.2 \mathrm{~Hz}\right) ; 16.5,16.3\left(2 \mathrm{~d}, 4 \mathrm{CH}_{3},{ }^{3} J_{C P} 6\right.$ $\left.\mathrm{Hz},{ }^{3} J_{C P} 6 . \mathrm{Hz}\right) ;{ }^{31} \mathrm{P}-\mathrm{NMR}\left(121 \mathrm{MHz}, \mathrm{CDCl}_{3}\right): 16.51$ (d, =CP, $J 25.4 \mathrm{~Hz}$ ); 22.24 (d, CHP, $J 25.4$ $\mathrm{Hz}$ ). $\mathrm{C}_{17} \mathrm{H}_{30} \mathrm{~N}_{2} \mathrm{O}_{6} \mathrm{P}_{2}$ : HRMS calculated for $\left[\mathrm{M}+\mathrm{H}^{+}\right]$421.16519, found 421.16476. 


\section{Acknowledgements}

This work was supported by the Higher Education and Scientific Research of Tunisia. The authors wish to thank professors Adel Nefzi (University of California San Diego) and Jacques Lebreton (University of Nantes, France) for logistical help.

\section{References}

1. Belaud, C.; Roussakis, C.; Letourneux, Y.; El Alami, N.; Villiéras, J. Synth. Commun. 1985, $15,1233$.

http://dx.doi/10.1080/00397918508077271

2. Villiéras, J.; Rambaud, M. Synthesis 1982, 924.

http://dx.doi/10.1055/s-1982-29998

3. Amri, H.; Rambaud, M.; Villiéras, J. J. Organomet. Chem. 1986, 308, C27. http://dx.doi.org/10.1016/S0022-328X(00)99740-7

4. Öhler, E.; Reininger, K.; Schmidt, M. Angew. Chem. Int. Ed. Engl. 1970, 9, 457. http://dx.doi/10.1002/anie.197004571

5. Knochel, P.; Normant, J. F. Tetrahedron Lett. 1984, 25, 1475. http://dx.doi.org/10.1016/S0040-4039(01)80190-8

6. Park, B. R.; Kim, K. H.; Lim, J. W.; Kim, J. N. Tetrahedron Lett. 2012, 53, 36. http://dx.doi/10.1016/j.tetlet.2011.11.001

7. Ben Ayed, T.; Amri, H.; El Gaïd, M. M. Tetrahedron 1991, 47, 9621. http://dx.doi.org/10.1016/S0040-4020(01)91028-2

8. György, K.; Imre, J.; István, G.; Mária, K. P.; János, B.; Lajos, S.; Csaba, S. J. Org. Chem. 1997, 62, 9188.

http://dx.doi/10.1021/jo9713464

9. Ben Ayed, T.; El Gaïd, M. M.; Amri, H. Synth. Commun. 1995, 25, 2981. http://dx.doi/10.1080/00397919508011429

10. Samarat, A.; Lebreton, J.; Amri, H. Synth. Commun. 2001, 31, 1675. http://dx.doi/10.1081/SCC100103986

11. Hoffmann, H. M. R.; Rabe, J. Angew. Chem. Int. Ed. Engl. 1983, 22, 795. http://dx.doi/10.1002/anie.198307951

12. Ben Ayed, T.; Amri, H.; El Gaïd, M.M.; Villiéras, J. J. Soc. Chim. 1992, III, 219.

13. Loh, T. P.; Lye, P. L. Tetrahedron Lett. 2001, 42, 3511. http://dx.doi/10.1016/S0040-4039(01)00492-0

14. Buchholz, R.; Hoffmann, H. M. R. Helv. Chim. Acta. 1991, 74, 1213. http://dx.doi/10.1002/hlca.19910740608

15. Paira, M.; Banerjee, B.; Jana, S.; Mandal, S. K.; Roy, S. C. Tetrahedron Lett. 2007, 48, 3205. http://dx.doi/10.1016/j.tetlet.2007.03.036

16. Szlosek-Pinaud, M.; Diaz, P.; Martinez, J.; Lamaty, F. Tetrahedron Lett. 2003, 44, 8657. 
http://dx.doi/10.1016/j.tetlet.2003.09.169

17. Ameer, F.; Drewes, S. E.; Emslie, N. D.; Kaye, P. T.; Mann, R. L. J. Chem. Soc., Perkin Trans. 1 1983, 28, 2293.

http://dx.doi/10.1039/P19830002293

18. Hoffmann, H. M. R.; Rabe, J. J. Org. Chem. 1985, 50, 3849.

http://dx.doi/10.1021/jo00220a034

19. Beltaïef, I.; Besbes, R.; Villiéras, J.; Amri, H. Tetrahedron Lett. 1997, 38, 813.

http://dx.doi.org/10.1016/S0040-4039(96)02437-9

20. Fray, A.; Ben Kraïem, J.; Souizi, A.; Amri, H. Arkivoc. 2012, viii, 119. http://dx.doi.org/10.3998/ark.5550190.0013.811

21. Kraïem, H.; Abdullah, I. M.; J.; Amri, H. Tetrahedron Lett. 2003, 44, 553. http://dx.doi.org/10.1016/S0040-4039(02)02589-3

22. Arfaoui, A.; Amri, H. Tetrahedron 2009, 65, 4904. http://dx.doi/10.1016/j.tet.2009.04.028

23. Kraïem, H.; Amri, H. Phosphorus, Sulfur and Silicon. 2007, 182, 2555. http://dx.doi/.org/10.1080/10426500701509329

24. Beltaïef, I.; Hbaïeb, S.; Besbes, R.; Amri, H.; Villiéras, M.; Villiéras, J. Synthesis 1998, 1765. http://dx.doi/10.1055/s-1998-2220

25. Rambaud, M.; Del Vecchio, A.; Villiéras, J. Synth. Commun. 1984, 14, 833. http://dx.doi/10.1080/00397918408075726

26. Basavaiah, D.; Pandiaraju, S. Tetrahedron 1996, 52, 2261. http://dx.doi.org/10.1016/0040-4020(95)01055-6

27. Das, B.; Bhunia, N.; Damodar, K. Synth. Commun. 2012, 42, 2479. http://dx.doi/10.1080/00397911.2011.561396

28. Pascual, C.; Meier, J.; Simon, W. Helv. Chim. Acta 1966, 49, 164. http://dx.doi/10.1002/hlca.660490122

29. Matter, U. E.; Pascual, C.; Pretsch, E.; Simon, W.; Sternhell, S. Tetrahedron 1969, 25, 691. http://dx.doi.org/10.1016/S0040-4020(01)83279-8

30. Béji, F.; Lebreton, J.; Villiéras, J.; Amri, H. Tetrahedron 2001, 57, 9959 and references cited herein. http://dx.doi.org/10.1016/S0040-4020(01)01018-3

31. Johannsen, M.; Jorgensen, K. A. Chem. Rev. 1998, 98, 1689. http://dx.doi/10.1021/cr9703430

32. Schlessinger, R. H.; Li, Y.-J. J. Am. Chem. Soc. 1996, 118, 3301. $\underline{\text { http://dx.doi/10.1021/ja954311z }}$

33. Tavares, F.; Lawson, J. P.; Meyers, A. I. J. Am. Chem. Soc. 1996, 118, 3303. http://dx.doi/10.1021/ja954312r

34. Entwistle, D. A.; Jordan. S. I.; Montgomery, J.; Pattenden, G. J. Chem. Soc., Perkin Trans. 1 1996, 1315. 


\section{http://dx.doi/10.1039/P19960001315}

35. Overman, L. E.; Petty, C. B.; Doedens, R. J. J. Org. Chem. 1979, 44, 4183. http://dx.doi/10.1021/jo01337a036

36. Bäckvall, J.-E.; Nordberg, R. E.; Nyström, J.-E.; Högberg, T.; Ulff, B. J. Org. Chem. 1981, $46,3479$. http://dx.doi/10.1021/jo00330a019

37. Stütz, A. Angew. Chem., Int. Ed. Engl. 1987, 26, 320. http://dx.doi/10.1002/anie.198703201

38. Cheikh, R. B.; Chaabouni, R.; Laurent, A.; Mison, P.; Nafti, A. Synthesis 1983, 685. http://dx.doi/10.1055/s-1983-30473

39. Hagithara, M.; Anthony, N. J.; Stout, T. J.; Clardy, J.; Schreiber, S. L. J. Am. Chem. Soc. 1992, 114, 6568. http://dx.doi/10.1021/ja00042a052

40. Devadder, S.; Verheyden, P.; Jaspers, H. C. M.; Van Binst, G.; Tourwé, D. Tetrahedron Lett. 1996, 37, 703.

41. Yammamoto, Y.; Schmid, M. J. Chem. Soc., Chem. Commun. 1989, 1310. http://dx.doi/10.1039/C39890001310

42. Yamaguchi, R.; Moriyasu, M.; Yoshioka, M.; Kawanisi, M. J. Org. Chem. 1985, 50, 287. http://dx.doi/10.1021/jo00202a031

43. Borzilleri, R. M.; Zheng, X.; Schmidt, R. J.; Johnson, J. A.; Kim, S. H.; DiMarco, J. D.; Fairchild, C. R.; Gougoutas, J. Z.; Lee, F. Y. F.; Long, B. H.; Vite, G. D. J. Am. Chem. Soc. 2000, 122, 8890. http://dx.doi/10.1021/ja001899n

44. Besada, P.; Mamedova, L.; Thomas, C. J.; Costanzi, S.; Jacobson, K. A. Org. Biomol. Chem. 2005, 3, 2016.

http://dx.doi/10.1039/B416349D

45. Jain, R. P.; Williams, R. M. J. Org. Chem. 2002, 67, 6361. http://dx.doi/10.1021/jo025636i

46. Felpin, F. X.; Girard, S.; Vo-Thanh, G.; Robins, R. J.; Villiéras, J.; Lebreton, J. J. Org. Chem. 2001, 66, 6305. http://dx.doi/10.1021/jo010386b

47. Ramachandran, P. V.; Burghardt, T. E.; Bland-Berry, L. J. Org. Chem. 2005, 70, 7911. http://dx.doi/10.1021/jo0508200 